\title{
Adverse event occurrence and treatment change in patients with schizophrenia; the GRACE study
}

\author{
Dimitra Karadima ${ }^{1 *}$, Ioannis Chatzimanolis ${ }^{2}$, Errikos Tsebelikos ${ }^{3}$, Venetsanos Mavreas ${ }^{4}$, Athanasios Fokas ${ }^{5}$, \\ Athanasios Kalogeropoulos ${ }^{6}$, Simeon Deres ${ }^{7}$, Theodosios Christodoulakis ${ }^{8}$ \\ From $1^{\text {st }}$ International Congress on Neurobiology and Clinical Psychopharmacology and European \\ Psychiatric Association Conference on Treatment Guidance \\ Thessaloniki, Greece. 19-22 November 2009
}

\section{Background}

Currently available, second generation antipsychotics interact with dopamine and serotonin receptors presenting less extrapyramidal symptoms and better tolerability in the elderly. Safety data gathered until today show that adverse events is one of the major reasons leading to antipsychotic treatment change, while in the meantime there is substantial differentiation of adverse events experienced by patients under treatment for schizophrenia.

The present analysis aims at assessing treatment changes made during the observation period of the study and record the frequencies of treatment change due to adverse event as well as other disease-related factors.

\section{Materials and methods}

The Grace study was a descriptive, cross-sectional, multicentre national survey conducted in the outpatient setting by 104 psychiatrists, proportionally distributed around Greece. Investigators registered one visit of each of the first 20 consecutive patients that presented at their consultation. Patient's demographics, living status, smoking status, alcohol consumption, blood laboratory values, and data on the reasons leading to treatment change during the past 6 months were recorded. Additionally, the reason and the antipsychotic drug selected to carry on with, after the last treatment change were also specified.

\section{Results}

The observational period was from June 2007 to June 2008 and led to the recruitment of 2013 patients with an ICD-10 primary diagnosis of schizophrenia. Out of them 523 were being administered with a pharmacological

${ }^{1}$ Bristol - Myers Squibb Greece treatment against schizophrenia. This population subset consisted of $51.6 \%$ men and $48.4 \%$ women, aged $39.9 \pm$ 12.4 years. The mean number of treatment changes during the past 6 months was $1.85 \pm 1.58$ times. According to the investigators, the major adverse events that lead to treatment change were extrapyramidal symptoms (44.7\%), weight gain (43.6\%) and suppression (25\%). For the most recent treatment change, apart from adverse event occurrence $(61 \%)$, other reasons that led to treatment change, concomitantly present or not with adverse events, were "no signs of improvement" (37\%) and "clinical deterioration" (21\%).

\section{Conclusions}

Adverse events occurrence remains an important reason for treatment change in schizophrenic patients treated with second generation antipsychotic agents. Extrapyramidal symptoms and weight gain are precarious adverse events that should alert physicians for their early recognition and management.

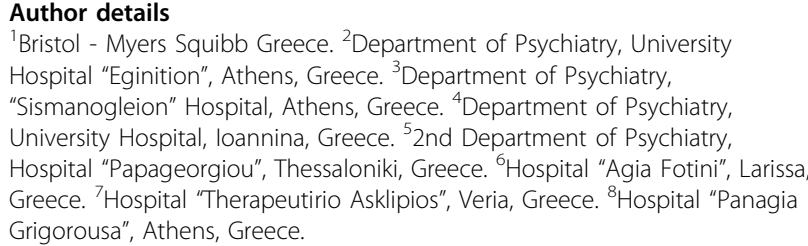

Published: 22 April 2010

doi:10.1186/1744-859X-9-S1-S94

Cite this article as: Karadima et al:: Adverse event occurrence and treatment change in patients with schizophrenia; the GRACE study. Annals of General Psychiatry 2010 9(Suppl 1):S94 